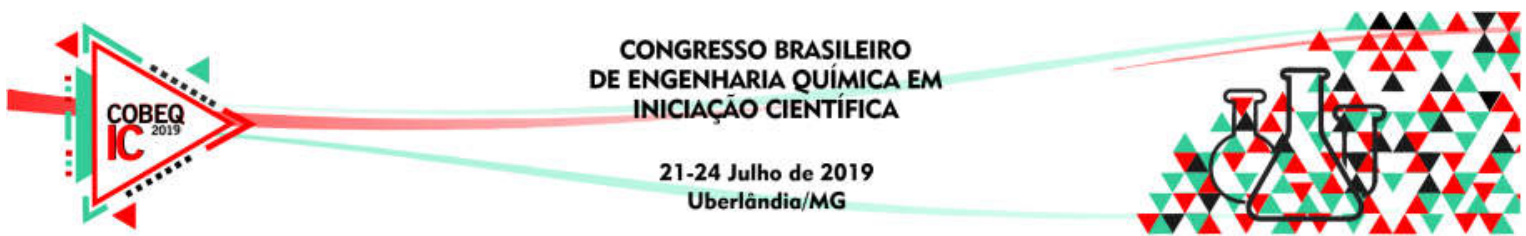

\title{
ESTUDO DE TRATABILIDADE EM ESCALA DE BANCADA PARA AVALIAR A EFICIÊNCIA DE DIFERENTES DOSES DE COAGULANTE NA ÁGUA DE UM MANANCIAL DA CIDADE DE OURO BRANCO/MG
}

\author{
NEVES T.C. ${ }^{1}$, BARBOSA A. ${ }^{1}$, DANIEL K.T. ${ }^{2}$, MELGAR L.Z. ${ }^{2}$ e SANTOS E.P.C.C. ${ }^{3}$ \\ ${ }^{1}$ Universidade Federal de São João Del-Rei, Engenharia Civil \\ ${ }^{2}$ Universidade de São João Del-Rei, Departamento de Engenharia Química \\ ${ }^{3}$ Universidade de São João Del-Rei, Departamento de Tecnologia em Engenharia de Civil, \\ computação e humanidades \\ E-mail para contato: eliane.santos@ufsj.edu.br
}

RESUMO - No presente trabalho foi realizado um estudo de tratabilidade para avaliar o desempenho de diferentes doses do coagulante sulfato de alumínio, na redução da cor aparente e da turbidez. Para o estudo foi utilizada a água do lago Soledade, localizado na cidade de Ouro Branco/MG, a qual é captada e tratada por meio do tratamento convencional por uma empresa da região. Para realizar o experimento foram coletadas catorze amostras, no período de junho a outubro de 2017. Todas as análises foram realizadas em duplicata e seguiram as prescrições do Standard Methods for the Examination of Water and Wastewater. Diante dos resultados obtidos nos ensaios em bancada, pôde-se observar que os melhores resultados de redução de turbidez e cor foram para a dose $30 \mathrm{mg}$. $\mathrm{L}^{-1}$. Os resultados mostraram que houve diferença significativa, considerando um nível de significância de $5 \%$, entre os valores de turbidez da água tratada com as doses de $30 \mathrm{mg} . \mathrm{L}^{-1}$ e $35 \mathrm{mg} . \mathrm{L}^{-1}, p=$ $4,33 \%$, ou seja, menor que $p_{0}=5 \%$.

\section{INTRODUÇÃO}

De acordo com relatório da UNESCO (2019), desde 1980 o uso dos recursos hídricos no mundo vem crescendo cerca de $1 \%$ ao ano. Isso está relacionado com o aumento populacional, o desenvolvimento sócio econômico e as mudanças no padrão de consumo. Essa demanda tende a continuar crescendo à mesma taxa até 2050, o que representa um aumento de 20 a 30\% em relação à demanda atual, principalmente devido ao aumento do consumo nos setores industrial e doméstico.

O acesso à água em quantidade e com qualidade é essencial tanto para prevenir doenças, quanto para produção industrial e agrícola. Para isso, é necessário que se protejam os mananciais e que se trate a água de forma que possa atender às necessidades do homem e aos padrões exigidos pelos órgãos fiscalizadores.

A água tratada para o consumo humano deve atender à Portaria consolidada $\mathrm{n}^{\mathrm{o}} 5$ de 2017 do Ministério da Saúde, que estabelece normas sobre as ações e os serviços de saúde do Sistema Único de Saúde. Já a qualidade da água para a indústria deve atender aos requisitos do processo de produção industrial envolvido e do produto a ser produzido. 


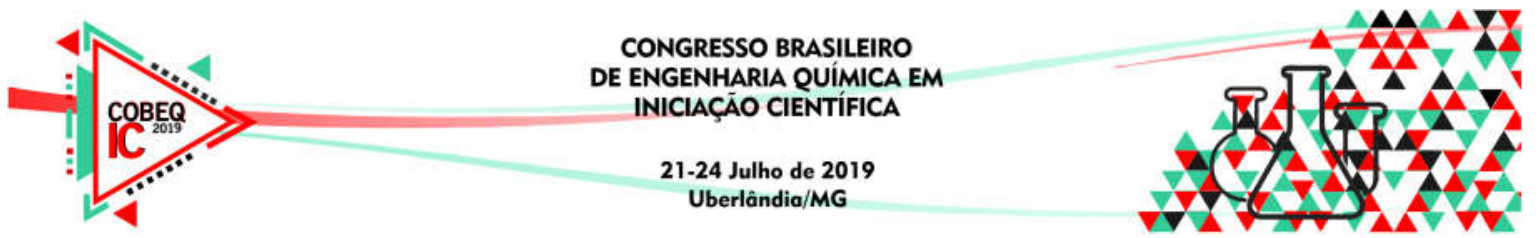

Quando se trata a água, independente da técnica de tratamento utilizada, há a formação de resíduos, os quais também devem ser tratados, a fim de proteger o meio ambiente e de atender à legislação vigente, especialmente às Leis $\mathrm{n}^{\circ} 9.433 / 1997, \mathrm{n}^{\circ}$ 9.605/1998 e a Resolução CONAMA no 237/1997. Entretanto, no Brasil, ainda há várias estações de tratamento de água cujos resíduos gerados são lançados sem tratamento em corpos d'água.

As características dos resíduos gerados durante o tratamento de água dependem: a) da técnica de tratamento utilizada para tratar a água; b) dos produtos utilizados durante o tratamento; c) da maneira como se operam e limpam as unidades de tratamento; d) da frequência de descarte dos resíduos; e) das características da água bruta e f) da vazão tratada e os locais onde é gerado o lodo, ou seja, no decantador, no floculador ou nos filtros.

Nos últimos anos, em diversos países, empresas que tratam a água vêm realizando estudos visando diminuir a dose e os tipos dos produtos utilizados para tratar a água, com objetivo de diminuir a quantidade de lodo gerado durante o tratamento de água.

De acordo a AWWA (1990), os dois componentes principais dos resíduos do tratamento de água (RTA) são os materiais contidos na água - solúveis ou insolúveis - e os produtos utilizados para a remoção destes materiais. As características químicas e físicas dos RTA variam conforme a composição química do coagulante. Consequentemente, problemas relacionados com o manuseio, o tratamento e a disposição dos RTA podem ser minimizados pelo ajuste do processo de coagulação e, em alguns casos, pela mudança do coagulante. Essas alterações têm contribuído para diminuir tanto o custo do tratamento de água quanto o volume dos resíduos gerados.

Diante disso, o presente trabalho teve como objetivo realizar um estudo de tratabilidade, comparando o desempenho de diferentes doses do coagulante sulfato de alumínio, na redução da cor aparente e da turbidez utilizando a água do lago Soledade na cidade de Ouro Branco/MG, a qual é captada e tratada por uma empresa na região.

\section{METODOLOGIA}

Para realizar o estudo foram coletadas amostras de água bruta do lago Soledade, manancial de onde uma empresa da região capta água para ser tratada pelo ciclo completo ou tratamento convencional e utiliza o coagulante testado. Foram coletadas catorze amostras no período de junho a outubro de 2017. Os parâmetros avaliados, tanto da água bruta, quanto da água tratada, por meio dos testes em bancada foram: cor aparente, turbidez e $\mathrm{pH}$. Todas as análises foram realizadas em duplicatas. A alcalinidade foi analisada somente para a água bruta, pois é um parâmetro cujo conhecimento é necessário para que se possa tratar a água com mais eficácia.

Todas as análises foram realizadas seguindo as prescrições do Standard Methods for the Examination of Water and Wastewater (AMERICAN PUBLIC HEALTH ASSOCIATION, 2005).

A Tabela 1 apresenta os equipamentos utilizados para as análises. Os ensaios foram realizados no laboratório de saneamento da Universidade Federal de São João del-Rei, campus Alto do Paraopeba, em Ouro Branco/MG. 


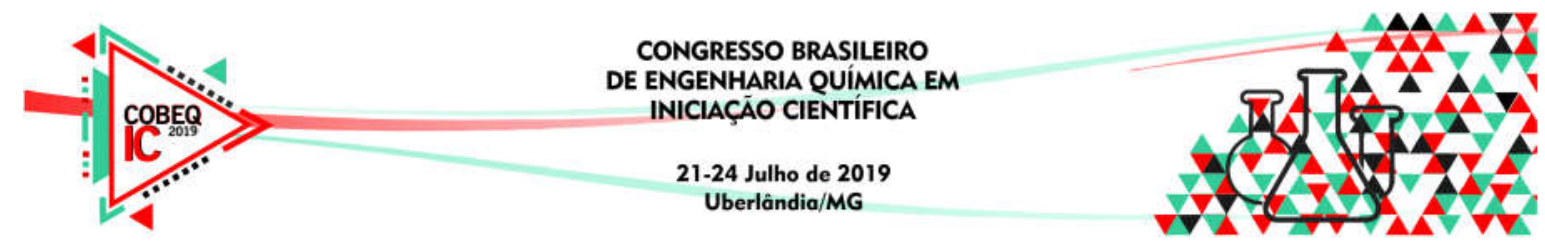

Tabela 1 - Equipamentos utilizados no estudo

\begin{tabular}{|c|c|}
\hline Equipamentos & Finalidade \\
\hline \hline Turbidímetro Digital Portátil Digimed-TU & para a quantificação da turbidez \\
\hline pHmetro Digimed DN22 & para medir o pH. \\
\hline Espectrofôtometro Geahaka modelo uis 200g & para quantificar a cor \\
\hline Jar-test Policontrol Floccontrol iii & para executar o estudo de tratabilidade \\
\hline
\end{tabular}

Para definir as doses dos coagulantes que seriam utilizadas no estudo foram realizados testes em bancada preliminarmente, avaliando diversas dosagens de coagulante. Após esses experimentos, definiu-se as seguintes doses, as quais foram usadas no estudo de tratabilidade: $35 ; 32,5 ; 30 ; 27,5$ e $25 \mathrm{mg}$. $\mathrm{L}^{-1}$. A tabela 2 apresenta os valores dos parâmetros utilizados no jar test durante os ensaios de tratabilidade.

Tabela 2 - Parâmetros utilizados nos ensaios de tratabilidade

\begin{tabular}{|c|c|c|}
\hline Etapa do tratamento & Gradiente de Velocidade $(\mathrm{G}=\mathrm{s}-1)$ & Tempo \\
\hline \hline Mistura rápida & 800 & 30 segundos \\
\hline Floculação & $120,100,80,60,40$ & 5 min para cada G usado \\
\hline Decantação & 20 & 25 min \\
\hline
\end{tabular}

\section{RESULTADOS E DISCUSSÃO}

A Figura 1 apresenta um gráfico de barras com os resultados de turbidez e de cor aparente da água bruta, obtidos durante os experimentos no período de junho a outubro de 2017. Como pode ser observado, os picos de cor aparente e de turbidez ocorreram simultaneamente. Nos dias em que foram observados os picos, houve uma grande intensidade de chuva na região, o que pode ter contribuído para esse aumento, pois é comum ocorrer o carreamento de partículas do solo para o corpo d'água.

Figura 1 - Gráfico de tempo $\times$ cor aparente e turbidez da água bruta.

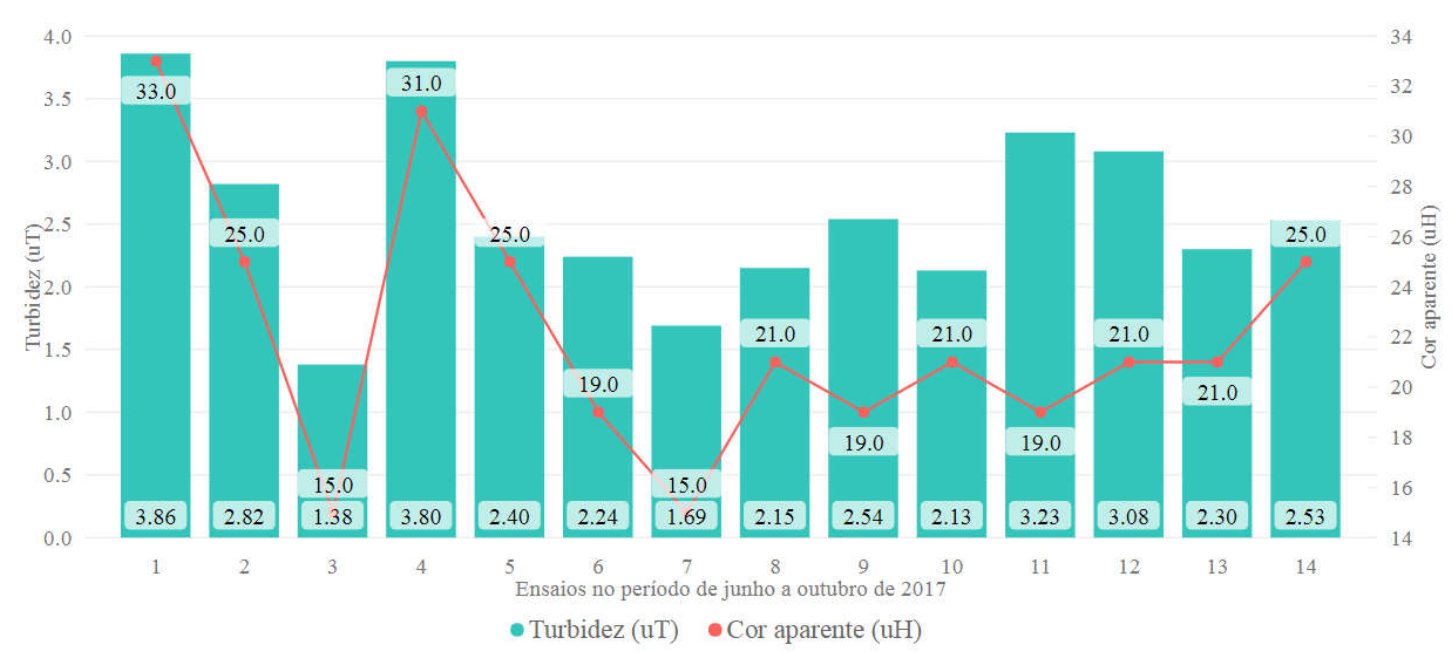

Vale ressaltar que os números dos eixos das abscissas das Figuras 1 e 2 se referem às amostras 1e 2 no mês de junho; amostras 3, 4 e 5 ao mês de agosto; amostras 6, 7, 8, 9 e 10 ao 


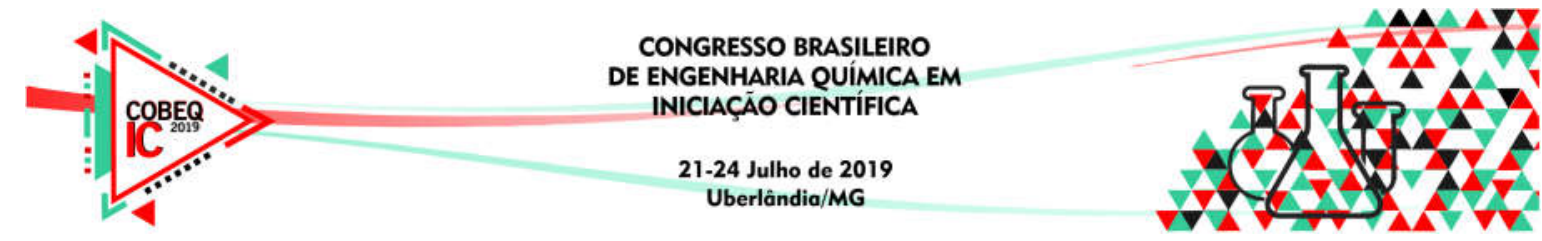

mês de setembro e as amostras 11, 12, 13, 14 ao mês outubro. Todas coletadas em 2017.

Observa-se que o maior pico de cor aparente ocorreu em junho (amostra 1), atingindo o valor de $33 \mathrm{uH}$, e que o segundo maior ocorreu no mês de agosto (amostra 4), com valor de $31 \mathrm{uH}$. Os valores de turbidez nesses meses foram a 3,86 uT e 3,80 uT, respectivamente.

A Figura 2 apresenta um gráfico de área mostrando os valores do $\mathrm{pH}$ da água bruta e do pH médio obtidos durante os experimentos no período de junho a outubro de 2017.

Figura 2 - Gráfico de tempo $\times \mathrm{pH}$ da água bruta.

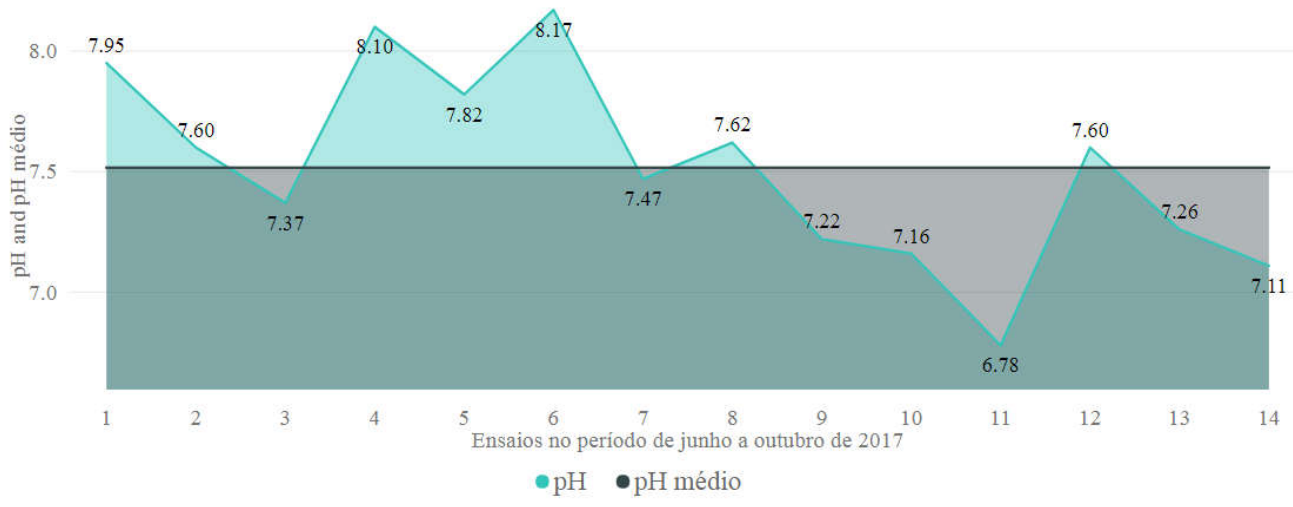

Observa-se na Figura 2 que os valores máximos de $\mathrm{pH}$ ocorreram em agosto (amostra 4) e em setembro (amostra 6), cujos valores foram de 8,10 e 8,17, respectivamente. Já o valor mínimo atingido pelo $\mathrm{pH}$ ocorreu em outubro, o qual foi de 6,78 (amostra 11). Já o valor médio do $\mathrm{pH}$ da água bruta no período do estudo foi de 7,52.

Para comparar os resultados obtidos nos ensaios com as respectivas doses testadas, considerou-se a média dos valores de turbidez e de cor aparente remanescentes e de $\mathrm{pH}$. Vale ressaltar que o valor da alcalinidade de água bruta variou de 40 a $50 \mathrm{mg} . \mathrm{L}^{-1} \mathrm{CaCO}_{3}$.

A Figura 3 apresenta os resultados obtidos de turbidez e remoção de turbidez para as diferentes doses de sulfato de alumínio testadas.

Figura 3 - Gráfico da concentração do Sulfato de Alumínio × Turbidez .

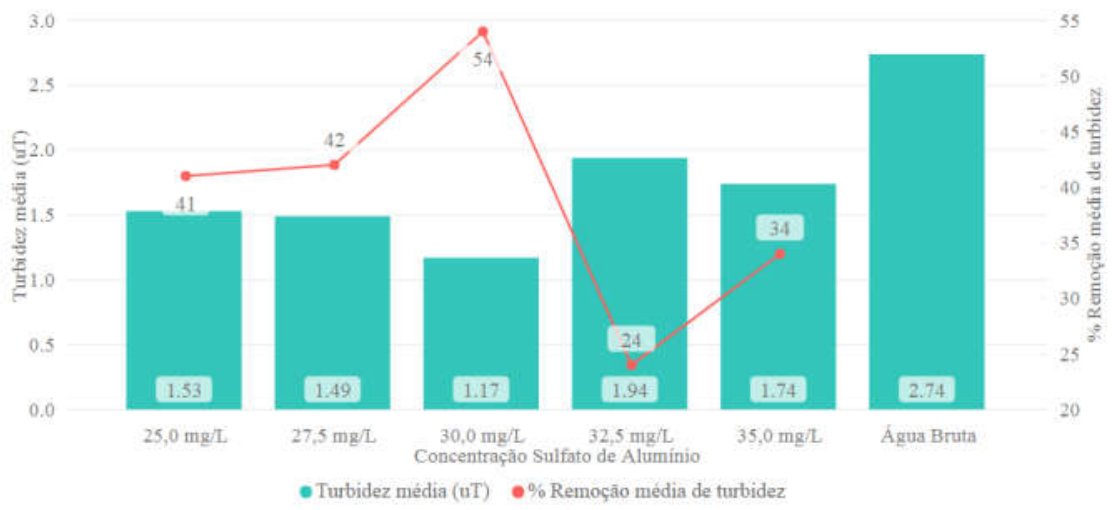




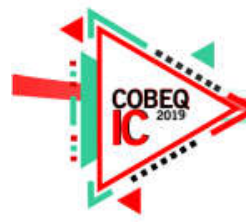

Observa-se, que o melhor desempenho do coagulante sulfato de alumínio foi para a dose de $30 \mathrm{mg}$. $\mathrm{L}^{-1}$, na qual se obteve o resultado para a turbidez média remanescente de 1,17 uT, e a porcentagem de redução média de turbidez em relação a água bruta foi de 54\%.

Já a Figura 4 apresenta os resultados obtidos de cor aparente e sua redução com as doses de sulfato de alumínio testadas.

Figura 4 - Gráfico da concentração do Sulfato de Alumínio × Cor aparente.

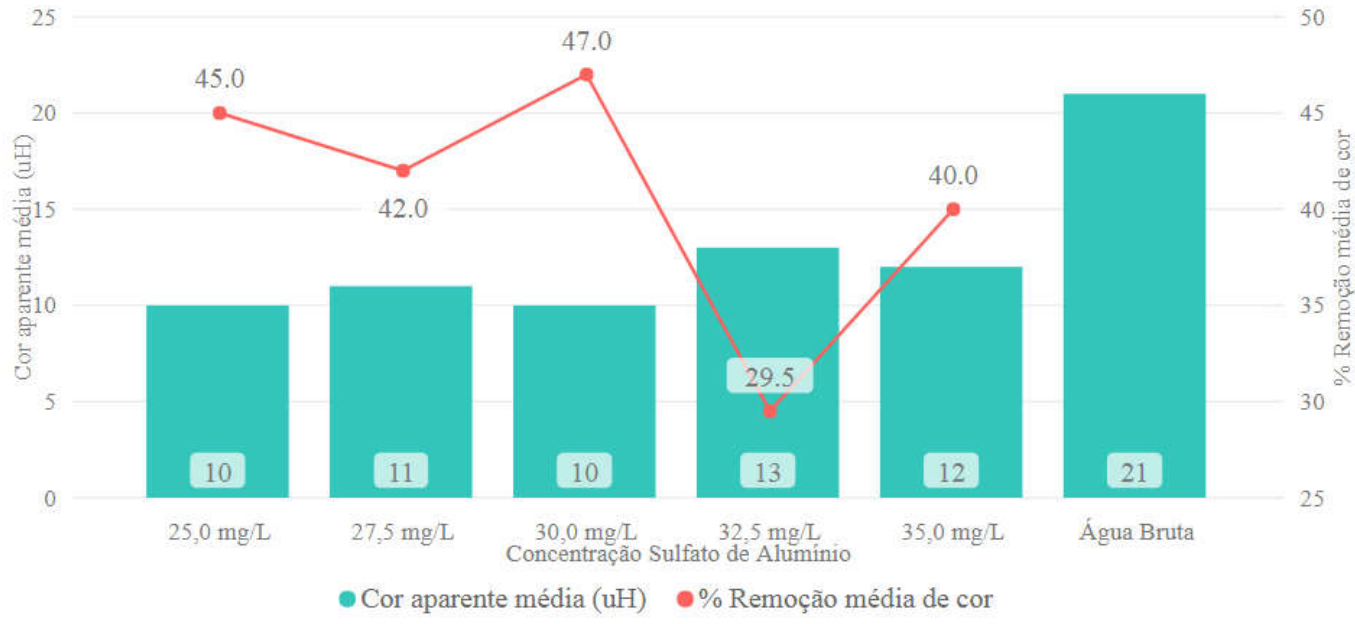

Com relação à cor aparente remanescente, observa-se que os melhores resultados foram para as doses de 25 e $30 \mathrm{mg}$. $\mathrm{L}^{-1}$, cujos valores foram de $10 \mathrm{uH}$. As porcentagens de redução média de cor para as doses de 25 e $30 \mathrm{mg}^{-\mathrm{L}^{-1}}$ foram de $45 \%$ e $47 \%$, respectivamente.

As Figura 5 e 6 mostram os gráficos comparativos entre os valores de turbidez e cor aparente das amostras de água tratada para as concentrações de $30 \mathrm{mg}$. $\mathrm{L}^{-1}$ (dose que proporcionou o menor valor de turbidez no estudo) e $35 \mathrm{mg} . \mathrm{L}^{-1}$ (dose usada pela estação de tratamento de água da empresa).

Figura 5 - Gráfico comparativo entre os valores de turbidez das amostras de água tratada para doses de $30 \mathrm{mg} . \mathrm{L}^{-1}$ e $35 \mathrm{mg}$. L $\mathrm{L}^{-1}$.

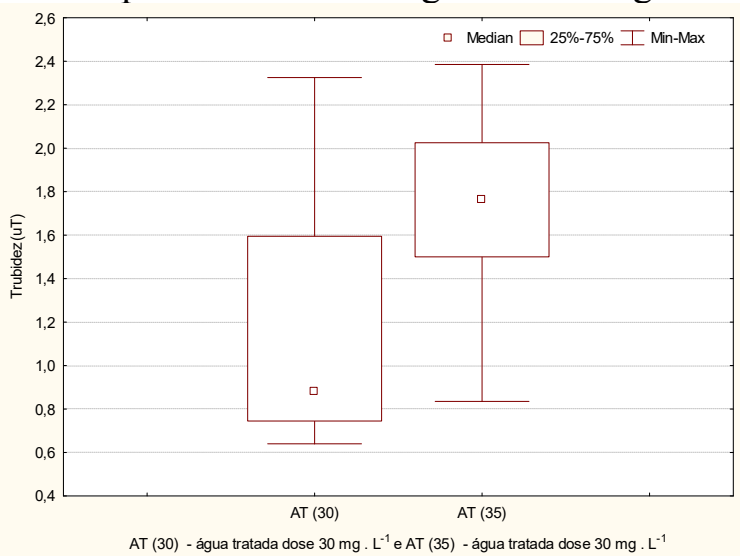

Figura 6 - Gráfico comparativo entre os valores de cor aparente das amostras de água tratada para doses de $30 \mathrm{mg} . \mathrm{L}^{-1}$ e $35 \mathrm{mg} . \mathrm{L}^{-1}$.

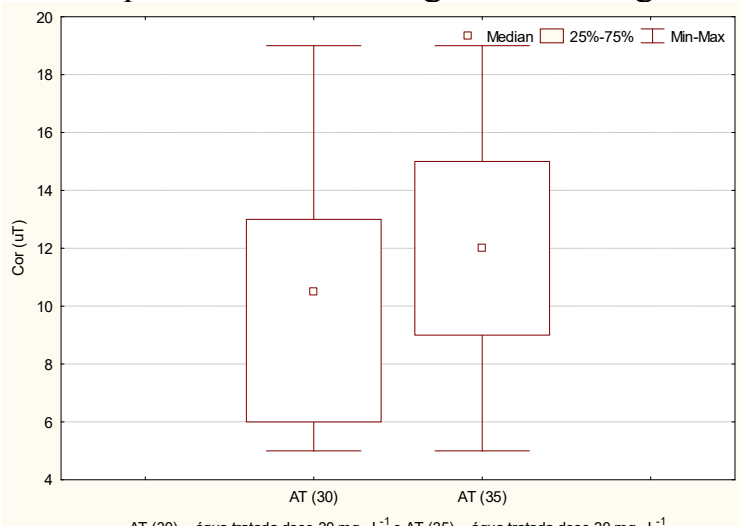




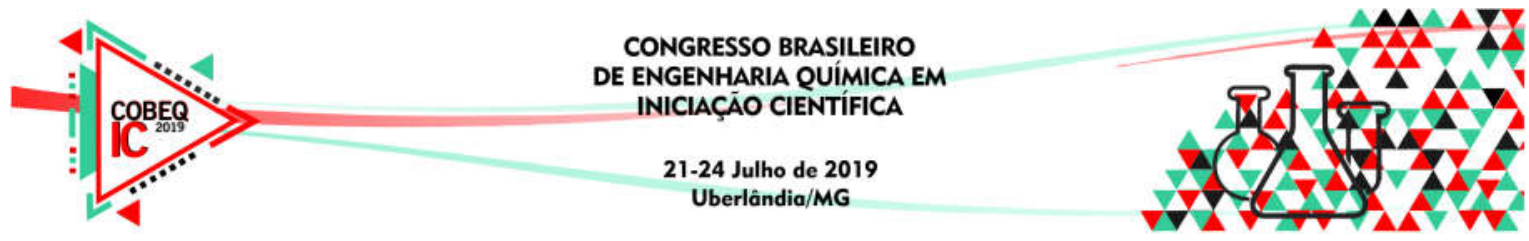

Houve diferença significativa, considerando um nível de significância de $5 \%$, para as comparações dos valores de turbidez remanescente das amostras de água tratada com as concentrações de $30 \mathrm{mg}$. $\mathrm{L}^{-1}$ e de $35 \mathrm{mg}$. L ${ }^{-1}$, sendo o valor do nível de significância de $4,33 \%$.

Não houve diferença significativa, considerando um nível de significância de $5 \%$, entre a cor remanescente da água tratada para as doses de $30 \mathrm{mg} . \mathrm{L}^{-1}$ e de $35 \mathrm{mg} . \mathrm{L}^{-1}$, sendo o valor do nível de significância de 50,01\%.

Vale ressaltar que no estudo de tratabilidade fazem-se análises dos parâmetros turbidez e cor remanescente da água decantada e no tratamento em escala real há ainda a filtração. Para a dose de coagulante de $30 \mathrm{mg} . \mathrm{L}^{-1}$ os valores da cor da água decantada já atenderia a legislação, pois todos os valores obtidos foram menores do limite de aceitação, ou seja $15 \mathrm{uH}$.

\section{CONCLUSÃO}

Diante dos resultados obtidos nos ensaios em bancada, pode-se observar que os melhores resultados de redução de turbidez e cor foram para a dose $30 \mathrm{mg}$. $\mathrm{L}^{-1}$.

As análises realizadas afirmam que não houve diferença significativa, considerando um nível de significância de $5 \%$, entre os valores de cor obtidos da água tratada com as doses de $30 \mathrm{mg} . \mathrm{L}^{-1}$ e de $35 \mathrm{mg} . \mathrm{L}^{-1}$, quando foram utilizados o coagulante sulfato de alumínio, sendo o nível de significância de 50,01\%.

Para os valores de turbidez da água tratada com as doses de $30 \mathrm{mg} . \mathrm{L}^{-1}$ e de $35 \mathrm{mg} . \mathrm{L}^{-1}$ houve diferença significativa $p=4,33 \%$ ou seja menor que $p_{0}=5 \%$.

\section{REFERÊNCIAS}

AMERICAN WATER WORKS ASSOCIATION. Water Quality and Treatment: $A$ Handbook of Community Water Supplies. 4th edition. New York: McGraw-Hill, Inc., 1990. $1194 \mathrm{p}$.

AMERICAN PUBLIC HEALTH ASSOCIATION. Standard Methods for the Examination of Water and Wastewater. 20. ed. Washington, DC: American Public Health Association, 2005.

CONAMA - Conselho Nacional do Meio Ambiente, 2011. Resolução nº430 de 13 de maio de 2011. Diário Oficial da União no 92, de 15 maio 2017.

MINISTÉRIO DA SAÚDE. Portaria Consolidada $n^{\circ}$ 5, de 28 de setembro de 2017. Consolidação das normas sobre as ações e os serviços de saúde do Sistema Único de Saúde. Diário Oficial da União $n^{\circ}$ 190, de 3 outubro 2017.

WWAP (UNESCO World Water Assessment Programme). 2019. The United Nations World Water Development Report 2019: Leaving No One Behind. Paris, UNESCO. 